\section{Cortical and spinal excitability}

Sir: I wish to comment on the report of reduced cortical excitability in depression (Shajahan et al, 1999).

First, the amount of time the subjects spent on exercising the muscle group was not stated. It is important for the exercise regime to be clearly stated so that the study can be replicated.

Second, it is believed that magnetic stimuli excite the cortex and the axon hillocks of the output neurons, whereas electrical stimuli directly excite corticospinal axons (Mills \& Kimiskidis, 1996). It follows that the size of electrically evoked responses will reflect predominantly spinal motorneuron excitability, whereas magnetically evoked responses will reflect both cortical and spinal excitability. Hallett (1996) indicates that it is possible to control for changes of excitability downstream from the cortical network, by using transcranial electrical stimulation, which stimulates the descending axons directly. Armand et al (1996) also show that if a corticospinal volley is evoked by transcranial magnetic stimulation (TMS), the electromyogram (EMG) response that results from it occurs after a certain delay including a central and peripheral conduction time. In most human studies, the central motor conduction time has been estimated by subtracting, from the latency of EMG response to TMS recorded in a given muscle, the latency of the responses excited by magnetic stimulation over the cervical spines. The latter is thought to excite the peripheral motor axons as they leave the vertebral column, and thus gives an estimate of the peripheral conduction time. Subtraction of this value allows the central motor conduction time to be calculated. In my opinion, the study design used by Shajahan et al is flawed as it fails to account for excitability at the spinal level.

Armand, J., Olivier, E., Ed,des S. A., et of (ISs) The structure and function of the developing corticospinal tract. In Hand and Broin: The Neurophysiology and Psychology of Hand Movement (eds A. M.Wing, P. Haggard \& J. R. Flanagan), pp. 125-145. London: Academic Press.

Halleta, M. (19\%) Transcranial magnetic stimulation: a useful tool for clinical neurophysiology. Annals of Neunology, 10, 344-345.

Muls, K. R. A KImidddi, V. (19\%) Motor cortex excitability during ballistic forearm and finger movements. Muscle and Nerve, 19, 468-473. Shajhan, P M, Ghabus, M. F, Cooding, P, ef ef (1999) Reduced cortical excitability in depression. Impaired postexercise motor facilitation with transcranial magnetic stimulation. British foumal of Pychiatry, 174, 449-454.

B.Y. Ng Department of Adult Psychiatry 2, Woodbridge Hospital and Institute of Mental Health, 10 Buangkok Green, Singapore 539747
Authors'reply: Dr $\mathrm{Ng}$ provides the very argument why post-exercise facilitation after transcranial magnetic stimulation (TMS) is a central rather than a peripheral phenomenon: transcranial electrically evoked responses reflect the stimulation of the descending axons only, while TMS acts trans-synaptically and by stimulating the axon near the cell body and is, therefore, dependent on the sum of all synaptic inputs to that neuron. As transcranial electrical stimulation demonstrates neither post-exercise facilitation nor depression, but TMS does, we are likely to be looking at central cortical excitability, rather than 'spinal excitability' (Samil et el, 1997). We have since shown that the reduced cortical excitability in depression is actually reversible with recovery from depression, independently of medication effects (Shajahan et al, 1999).

Exercise during the experiments was at $20 \%$ of maximum voluntary contraction for $30 \mathrm{~s}$ at a time. The procedure was repeated altogether five times. Any further details of the experimental set-up are available upon request from the authors.

Samil, A., Waseermann, E. M. \& Hallett, M. (I9)7) Decreased postexercise facilitation of motor evoked potentials in patients with cerebellar degeneration. Neurology, 49, 538-542.

Shaluhan, P. M., Chabus, M. E., Jenidins, J, et of (1999) Post-exercise motor evoked potentials in depressed, recovered depressed and controls. Neurology, in press.

P. Shajahan, M. Glabus, K. Ebmeler MRC Brain Metabolism Unit, Royal Edinburgh Hospital, Morningside Park, Edinburgh EHIO 5HF

\section{Ethnicity and first year of contact with psychiatric services}

Sir: The reports of Goater et al (1999) and Harrison et al (1999) seem to converge on the point that the course and outcome of first-onset psychotic illness are not significantly different in White and AfricanCaribbean populations in Britain. In both papers, the authors wisely caution against being too conclusive regarding their findings because of the small size of their respective samples.

In spite of the final consistency of the symptoms and diagnoses at the end of the follow-up periods, it is clear that the first year is central to the future interaction of the patients of African-Caribbean origin with psychiatric services. After one year, there were large relative increases in both psychotic and non-psychotic symptoms for the Black Caribbean patients and no convincing explanation is offered for this escalation of psychopathology that occurs during the first year of contact. The relationship between appropriateness and adequacy of treatment, compliance and phenomenology clearly needs to be investigated further. Goater et al (1999) suggest the increasing rate of conversion to schizophrenia and poorer compliance as plausible reasons for the adverse contacts that the Black patients have with psychiatric services in the fifth year of follow-up, in spite of the similar course. However, with similar modes of entry at first contact, it is likely that poor compliance would be a consequence rather than a cause of these problems. Both papers also point to a trend for a greater rate of changes in diagnoses among the African-Caribbean patients with the tendency to conversion to schizophrenia being strongest. Therefore, one wonders whether the experience of the service itself is schizophrenogenic for Black patients and, therefore, invites the future perceptions of abnormalities in speech, affect and behaviour which are more likely to meet criteria for schizophrenia and require more coercive means of management.

Research now needs to focus on the course of therapeutic intervention as generic approaches of management of psychotic illness may need to be reviewed to include both non-psychotic and psychotic symptoms. Comparisons with White British patients may not be entirely useful in these circumstances. This is reflected in the relative instability of both 'other' and 'schizophrenia' diagnoses for the Black patients compared with the White/Other groups. Finally, demographic categories need to be carefully constructed so that within-group differences can be examined more thoroughly.

Coater, N., King, M. Cole, E., et of (IS\%) Ethnicity and outcome of psychosis. British fournd of Psychiotry. 175, 34-42.

Harricon, G., Amln, S., Singh, S. P., et ol (1999) Outcome of psychosis in people of African-Caribbean family origin. Population-based first-episode study. British journal of Psychiatry, 175, 43-49.

G. Hutchinson, M. Sharpley Social Psychiatry Section, Institute of Psychiatry, De Crespigny Park, Denmark Hill, London SE5 8AF

\section{Psychiatry and homosexuality}

Sir: King \& Bartlett's (1999) review is both frank and commendable. They rightly 